\title{
A comparison of the nutritional quality of gluten free and mixed diets in UK adults
}

\author{
Z. Sobocinska, T.J. Butler and L. O’Connor \\ Department of Health Professions, Manchester Metropolitan University, Manchester, M15 6BG
}

Gluten-free diets (GFD), which are prescribed to patients diagnosed with a coeliac disease, have become an attractive choice among certain populations. In the US, a three-fold increase in prevalence of people without coeliac disease following GFD in a recent 5 year period has been reported ${ }^{(1)}$. However, the nutritional quality of a GFD has not been widely researched. Thus, the aim of this study was to compare the nutritional quality of the diets of UK adults consuming GFD and, mixed diets (no gluten restrictions).

Participants were recruited through social media. Questionnaires were posted and stamped addressed envelopes provided to facilitate questionnaire return. Data on socio-demographics, coeliac disease diagnosis and reasons for following a GFD were collected using a general health questionnaire. Dietary intakes were collected using the EPIC-Norfolk FFQ and converted into food and nutrient intakes using FETA ${ }^{(2)}$. The nutritional composition of gluten containing foods in the FFQ were adjusted to reflect the nutritional composition of gluten free alternatives for participants following GFD. Mean daily intake, from food only, of energy, macronutrient, fibre (NSP), micronutrient and food groups of those consuming GFD and mixed diets and, by reason for following GFD, were compared using independent t-tests.

69 questionnaires were returned and used in analyses (Mixed diet, $n=24$; GFD, $n=45$ ). Participants were largely women (mixed diet, $70 \%$; GFD, $91 \%$ ). There was no difference in age by diet type (Mixed diets, $38 \pm 12$ years; GFD, $42 \pm 14$ years, $p=0 \cdot 304$ ). Those that consumed a mixed diet had a higher mean age of finishing fulltime education ( $24 \pm 7$ years) than those that consumed a GFD (20 \pm 6 years), $\mathrm{p}=0.039$. The reasons given for following a GFD were: Coeliac disease $(53 \%)$, intolerance $(22 \%)$, Irritable Bowel Syndrome (6\%), allergy $(9 \%)$ and other $(9 \%)$. Intakes of energy $(\mathrm{kJ})$, macronutrient $(\mathrm{g} / \mathrm{d}, \%$ total energy) and fibre $(\mathrm{g} / \mathrm{d})$ were not different in those that consumed mixed diets or GFD $(\mathrm{p}>0.05)$. Intakes of most micronutrients examined were higher in those that consumed mixed diets than GFD (Table 1). Milk intakes were higher in those that consumed mixed diets (mean \pm SD: $336 \pm 179 \mathrm{~g} / \mathrm{d}$ ) than in those who consumed GFD (mean \pm SD: $74 \pm 95 \mathrm{~g} / \mathrm{d}$ ), $\mathrm{p}<0.001$. There were no other significant differences in food group intakes by diet type. There were no significant differences in dietary intakes by reason for following GFD [dichotomised as Coeliac disease, $n=24$; personal choice, $n=21$ ].

Table 1. Comparison of micronutrient intakes in participants consuming gluten free diets and mixed diets

\begin{tabular}{|c|c|c|c|c|c|c|c|c|c|c|c|}
\hline & \multicolumn{2}{|c|}{$\begin{array}{l}\text { Mixed diets } \\
(n=27)\end{array}$} & \multicolumn{2}{|c|}{$\begin{array}{l}\text { Gluten free diets } \\
(\mathrm{n}=45)\end{array}$} & \multirow[b]{2}{*}{ p-value* } & & \multicolumn{2}{|c|}{$\begin{array}{l}\text { Mixed diets } \\
(n=27)\end{array}$} & \multicolumn{2}{|c|}{$\begin{array}{l}\text { Gluten free diets } \\
\quad(\mathrm{n}=45)\end{array}$} & \multirow[b]{2}{*}{ p-value* } \\
\hline & $\overline{\text { Mean }}$ & $S D$ & $\overline{\text { Mean }}$ & $S D$ & & & $\overline{\text { Mean }}$ & $S D$ & Mean & $S D$ & \\
\hline Calcium (mg) & 923 & 362 & 566 & 297 & $<0.001$ & Folate $(\mu \mathrm{g})$ & 304 & 111 & 257 & 109 & 0.096 \\
\hline Potassium (mg) & 3714 & 970 & 3103 & 1215 & 0.037 & Niacin (mg) & $24 \cdot 7$ & $8 \cdot 1$ & $20 \cdot 9$ & $8 \cdot 3$ & $0 \cdot 072$ \\
\hline Magnesium (mg) & 340 & 103 & 276 & 116 & $0 \cdot 027$ & Riboflavin (mg) & 2 & $0 \cdot 7$ & $1 \cdot 2$ & $0 \cdot 5$ & $<0.001$ \\
\hline Phosphorus (mg) & 1473 & 452 & 1102 & 414 & $0 \cdot 001$ & Thiamin (mg) & 1.5 & $0 \cdot 4$ & $1 \cdot 2$ & $0 \cdot 4$ & $0 \cdot 013$ \\
\hline Sodium (mg) & 2885 & 1133 & 2331 & 946 & $0 \cdot 034$ & Cobalamin $(\mu \mathrm{g})$ & $7 \cdot 8$ & $5 \cdot 1$ & $5 \cdot 4$ & $3 \cdot 3$ & $0 \cdot 021$ \\
\hline Iron $(\mathrm{mg})$ & $11 \cdot 5$ & $3 \cdot 4$ & $9 \cdot 6$ & $3 \cdot 4$ & 0.024 & Pyridoxine (mg) & $2 \cdot 2$ & 0.7 & 1.9 & 0.7 & 0.033 \\
\hline Iodine $(\mu \mathrm{g})$ & 157 & 65 & 112 & 68 & $0 \cdot 01$ & Carotene $(\mu \mathrm{g})$ & 4439 & 2405 & 4572 & 2838 & $0 \cdot 845$ \\
\hline Copper (mg) & $1 \cdot 2$ & 0.5 & $1 \cdot 2$ & $0 \cdot 5$ & 0.565 & Vitamin E (mg) & $12 \cdot 1$ & 4.9 & $10 \cdot 9$ & 4.9 & $0 \cdot 311$ \\
\hline Zinc (mg) & $9 \cdot 6$ & 3 & $7 \cdot 7$ & $2 \cdot 7$ & 0.009 & & \multicolumn{2}{|c|}{ Median $(I Q R)$} & \multicolumn{2}{|c|}{ Median $(I Q R)$} & \\
\hline Manganese (mg) & $3 \cdot 7$ & 1.4 & $3 \cdot 3$ & 1.6 & $0 \cdot 26$ & Vitamin C (mg) & 133 & 82,147 & 111 & 73,152 & $0.474 * *$ \\
\hline Selenium $(\mu \mathrm{g})$ & $70 \cdot 7$ & $35 \cdot 5$ & $58 \cdot 4$ & $26 \cdot 1$ & $0 \cdot 107$ & Vitamin D $(\mu \mathrm{g})$ & $2 \cdot 6$ & $2 \cdot 1,5 \cdot 3$ & $2 \cdot 4$ & $2 \cdot 41 \cdot 5,2 \cdot 9$ & $0.045^{* *}$ \\
\hline
\end{tabular}

*as calculated using an independent samples t-test. **estimated using log transformed intakes.

In conclusion, exclusion of gluten from the diet may be contributing to low micronutrient intakes. Public health strategies to increase micronutrient intake in those that consume a GFD and to dissuade restriction of diets unnecessarily should be considered.

1. Kim H, Patel KG, Orosz E et al. (2016) JAMA Inter Med 176, 1716-1717.

2. Mulligan AA, Luben RN, Bhaniani A et al. (2014) BMJ Open 4, p.e004503. 